\title{
Application of Spatial and Feature Analysis to Electron Microscope Petrography.
}

\author{
J.S. Lowther and A.P. Wisher
}

Geology Department, University of Puget Sound, 1500 N. Warner \#1048, Tacoma, WA 98416-1048

A common procedure in the laboratory analysis of rocks is to prepare polished cross-sections of the specimens and to examine these sections in a scanning electron microscope equipped with a good backscattered (BSE) detector. This produces gray-scale images of the different rock components, usually minerals, based on their atomic-number differences. This in turn permits the determination of the chemical composition of each of the components by microbeam analysis. There is also a wealth of petrographic information in the BSE images themselves, which can be gathered using techniques of image processing and image analysis.

The BSE images are captured on film and digitized with a film scanner, or are taken directly into the computer with an analog-digital converter. Image-processing techniques can then be applied to enhance the characteristics (e.g., the size, shape, and arrangement of component grains) of the rock or of any part of it. We prefer Adobe Photoshop ${ }^{\mathrm{TM}}$ to process the images but there are many other software packages, such as NIH Image ${ }^{\mathrm{TM}}$, which work very well. We most commonly use the software to adjust contrast/brightness, to do thresholding, and to convert the gray-scale to a color scale. Analysis of processed images can be carried out with software which does gray-level (or color) histograms, "particle analysis", pixel counts on binary images, or stereology grids.

We have found it especially useful to capture a series of overlapping images and to assemble them into one continuous image in the same way as we would do with vertical aerial photographs. We can do strips by taking images along the " $\mathrm{X}$ " or " $\mathrm{Y}$ " direction or mosaics of images taken along both directions. We prefer to allow about $40 \%$ overlap between adjacent frames, either horizontally or vertically. There are several programs, including Photoshop, which can be used to assemble (stitch) together such strips or mosaics.

These procedures are applicable to any type of rock. We have gained some unique micropetrographic and microstratigraphic information by applying this stitching technique to images of a section of turbidites (turbidity-current deposited graded beds). cut perpendicular to the stratification (FIG.1 \& 2) The strip is long enough and with sufficiently high magnification to show more than one complete graded bed and the detailed relationships of the contacts between individual graded beds. A section of a lava bed cut perpendicular to the cooling surface and extending from the glassy crust down to the wholly crystalline lower part shows how the rock has solidified and crystallized and the order in which the individual minerals have formed. We have also assembled a strip section of an unusual Kimberlite with fragmental texture (FIG. 3). Additional analyses can be performed with a coordinate-based system like ArcView ${ }^{\mathrm{TM}}$. A spatial framework is setup in ArcView $^{\mathrm{TM}}$ to perform basic analysis including cell statistics, neighborhood statistics, and zonal statistics. Bins of similar gray values can be used to create polygons, which are dissolved and used for analysis. Additionally, the created polygons are used to build a database of chemical data and statistical data. Having the chemical data referenced spatially to the image allows for chemical relationships to be easily visualized. 

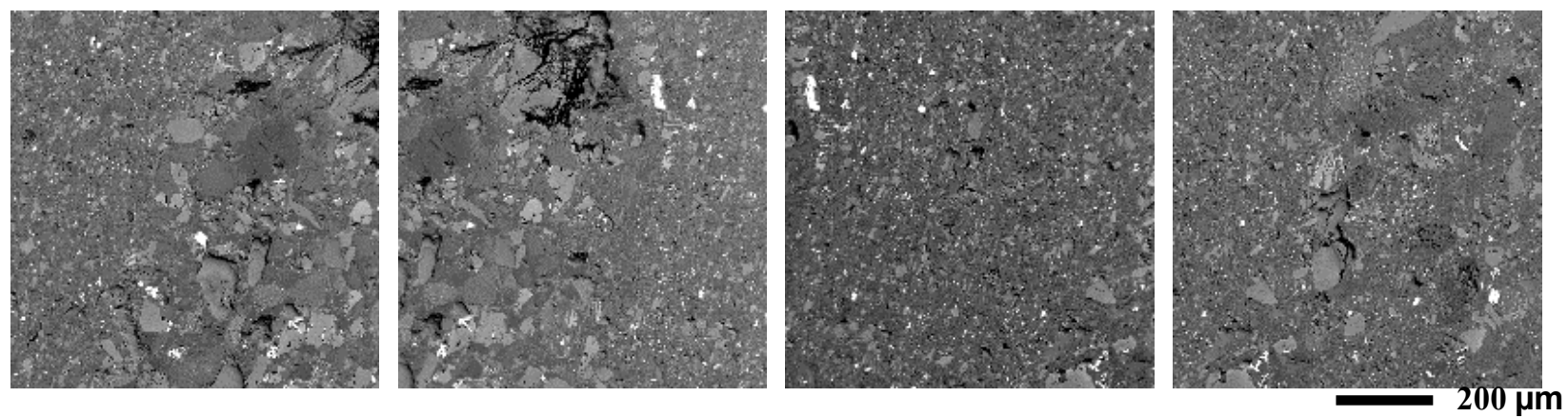

FIG. 1 Four overlapping BSE images of a basaltic sandstone turbidite.

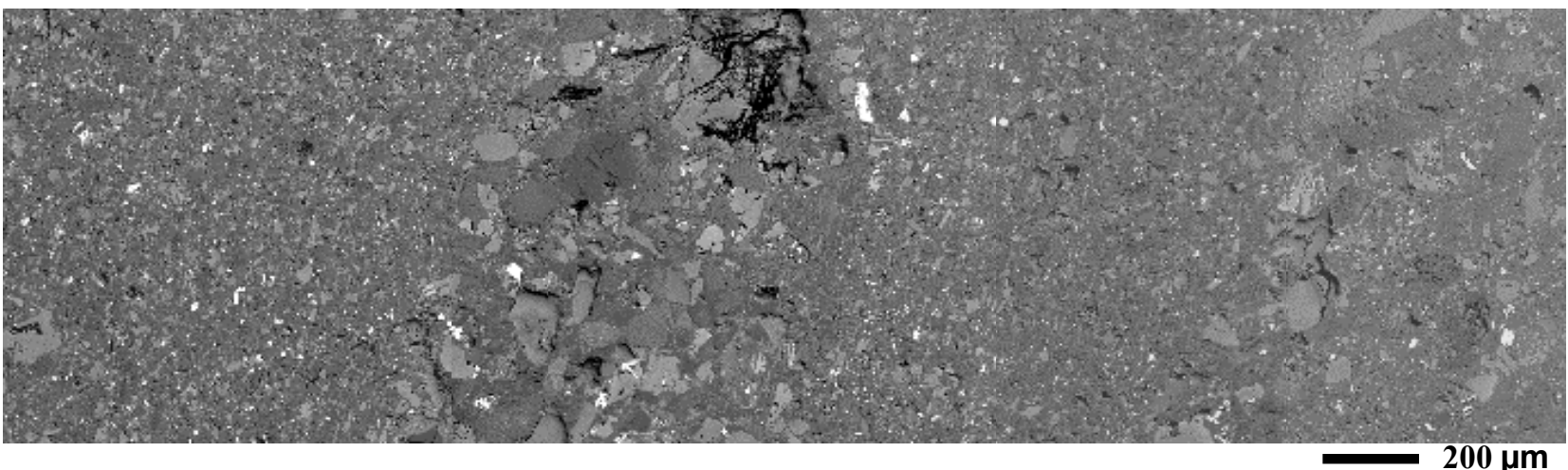

FIG. 2 Strip mosaic incorporating images in FIG. 1 showing two graded beds. Gray levels represent mineral components- dark plagioclase, intermediate pyroxenes, and white $\mathrm{Fe} / \mathrm{Ti}$ oxides.

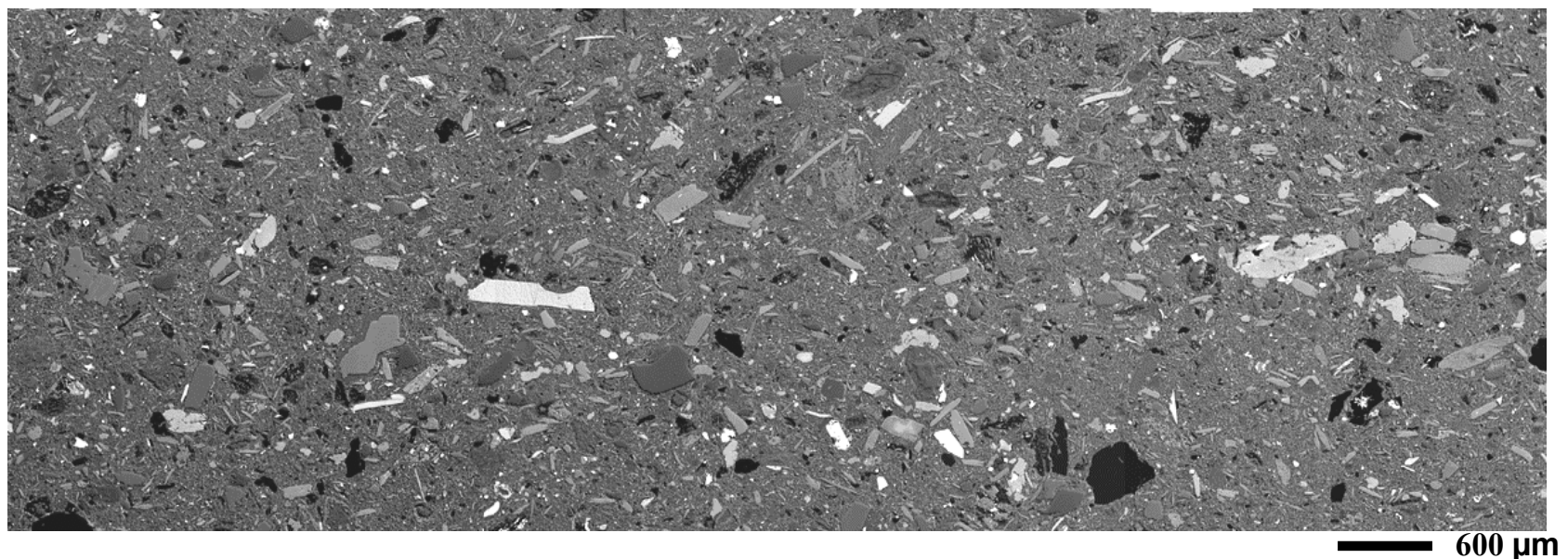

FIG. 3 Strip mosaic of a clastic-textured kimberlite. 